\title{
Coordenação motora de crianças e adolescentes portadores do vírus da imunodeficiência humana
}

\author{
Motor coordination of children and adolescents with human immunodeficiency virus
}

Karina Carvalho Marquesa, Chrisna Mayra de Brito Barbosa Abdonª, Liliane Bitencourt Santa Mariaa, Saul Rassy Carneirob , Márcio Clementino dos Santosc, Valéria Marques Ferreira Normando ${ }^{d}$, George Alberto da Silva Diasc, Erica Feio Carneiro Nunese, Lucieny da Silva Pontes ${ }^{f}$

a Fisioterapeuta Graduada pela Universidade da Amazônia (UNAMA), Brasil.

b Fisioterapeuta. Doutorando do Programa de Pós-graduação em Doenças Tropicais da Universidade Federal do Pará (UFPA), Brasil.

c Fisioterapeuta. Doutor em Doenças Tropicais pela UFPA. Professor da Universidade do Estado do Pará (UEPA), Brasil.

d Fisioterapeuta. Doutora em Neurociências e Biologia Celular pela UFPA, Brasil.

e Fisioterapeuta. Mestre em Ciências da Motricidade Humana. Professora da UEPA, Brasil.

Fisioterapeuta. Doutoranda do Programa de Pós-graduação em Doenças Tropicais da UFPA. Professora da UEPA, Brasil.

Fontes de financiamento: Bolsa de iniciação científica pela Fundação Instituto para o Desenvolvimento da Amazônia (FIDESA).

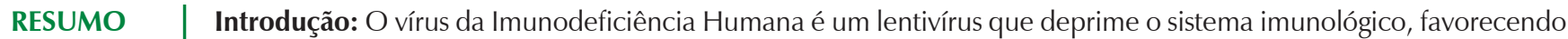
o desencadeamento de déficits, incluindo no sistema motor. Esses déficits podem ser gerados através de efeitos adversos da terapia antirretroviral.

Objetivos: Descrever o perfil da coordenação motora de crianças e adolescentes infectadas por via vertical pelo vírus da Imunodeficiência Humana e expostas ao uso de terapias antirretrovirais.

Materiais e Métodos: Estudo do tipo observacional e transversal, onde foram avaliadas 34 crianças e adolescentes matriculadas na Unidade de Referência Materno Infantil e Adolescente do Pará e distribuídas em dois grupos: 1) Grupo Controle: $n=13$, com sorologia negativa para o vírus da imunodeficiência humana, porém expostos a medicação no pré, peri ou pós-natal; 2) Grupo Experimental: $n=21$, crianças com sorologia positiva para o HIV e exposição prolongada as terapias antirretrovirais há mais de 1 ano. Foi utilizada a bateria de teste Korperkoordination test fur Kinder para análise do desempenho motor. A análise estatística foi realizada pelo software Bioestat ${ }^{\circledR}$ 5.0, considerando nível de significância de $5 \%$.

Resultados: Identificou-se no grupo experimental déficit na coordenação motora para tarefas de equilíbrio $(p=0,003)$ e lateralização $(p=0,007)$ comprometendo a conscientização corporal. A análise do quociente motor global e deste com relação a idade cronológica também apresentaram significância, respectivamente $p=0,027$ e $p=0,003$.

Conclusão: Crianças e adolescentes infectadas por via vertical pelo vírus da Imunodeficiência Humana e expostas ao uso prolongado da terapia antirretroviral apresentaram comprometimento do desempenho motor para as tarefas de equilíbrio e lateralidade.

Palavras-chave: antígenos HIV; desenvolvimento infantil; sorodiagnóstico da aids; terapia antirretroviral de alta atividade.

Introduction: The Human Immunodeficiency Virus (HIV) is a lentivirus that depresses the immune system, favoring the triggering of deficits, including the motor system. These deficits can be generated through the adverse effects of antiretroviral therapy.

Objectives: To describe the motor coordination profile of children and adolescents infected by the HIV and exposed to the use of antiretroviral therapies.

Materials and Methods: A cross-sectional, observational study was carried out, in which 34 children and adolescents enrolled in the Maternal and Child Referral Unit of Pará were divided into two groups: 1) Control Group: $n=13$, with negative HIV serology, but exposed to pre, peri or postnatal medication; and 2) Experimental group: $n=21$, children seropositive for HIV and prolonged exposure to antiretroviral therapies for more than 1 year. The Korperkoordination test fur Kinder test battery was used for motor performance analysis. Statistical analysis was performed using Bioestat ${ }^{\circledR} 5.0$ software, considering a significance level of $5 \%$.

Results: We identified motor coordination deficit for tasks of balance $(p=0.003)$ and lateralization $(p=0.007)$ compromising the body awareness in the experimental group. The analysis of the global motor quotient and of this with chronological age were also significant, respectively $p=0.027$ e $p=0.003$.

Conclusion: Children and adolescents infected vertically with the HIV and exposed to antiretroviral therapy had motor performance impairment for the tasks of balance and laterality.

Keywords: HIV antigens; child development; aids serodiagnosis; antirretroviral therapy, highly active. 


\section{INTRODUÇÃO}

A síndrome da imunodeficiência adquirida (sigla em inglês, AIDS) é considerada um dos maiores problemas de saúde pública do mundo contemporâneo, uma vez que se trata de uma doença grave ${ }^{1}$.

A AIDS vem assombrando o Brasil e o mundo há mais de três décadas. No mundo, estima-se que no ano de 2012 havia 35,3 milhões de pessoas infectadas pelo vírus da imunodeficiência humana (HIV) somando-se a 2,3 milhões de casos novos. Ainda neste cenário, cerca de 1,6 milhões morreram com AIDS em 2012, por dificuldade de acesso aos serviços de saúde². Até junho de 2015 foi possível registrar, no Brasil, 798.366 casos de AIDS e, ainda, detectou-se tendência de crescimento nos últimos dez anos de mulheres grávidas infectadas pelo HIV. Pressupõe-se que mais de 7 mil pessoas sejam infectadas diariamente, o que faz da AIDS um grande desafio para a gestão da saúde pública ${ }^{2,3}$.

Diante do quadro alarmante de infecção pelo vírus HIV em mulheres, a transmissão vertical (materno-fetal) vem se tornando uma preocupação no âmbito da saúde, sendo responsável por $84 \%$ dos casos. No Brasil, porém, a taxa de transmissão vertical vem decrescendo em função do uso de terapia antirretroviral (TARV) a gestantes e crianças expostas nos períodos pré, peri e pós-natal ${ }^{4}$.

Em relação a números absolutos, a região Norte apresenta taxa de incidência de AIDS de 17,6/100.00 habitantes. O maior número está no Pará, com 12.532 casos. Foi observado em 2010 aumento significativo na taxa de incidência em crianças de 5 a 12 anos, e quanto aos menores de 5 anos, entre 1980 e 2014, foram registrados 7.680 casos na Região Norte $^{5}$

Segundo dados fornecidos pela Unidade de Referência Materno Infantil e Adolescente (URE-MIA, Belém-PA), em fevereiro de 2016 foram registradas: a) 224 crianças com AIDS em uso de TARV; b) 574 crianças foram submetidas à TARV durante a gestação: c) 609 casos de crianças também submetidas à TARV, mas no período pós-natal: d) 52 crianças com sorologia positiva para HIV: e) 681 casos sem diagnóstico definido. Ocorreram 62 óbitos, no total.

Os avanços da TARV de primeira linha resultaram em redução expressiva da morbidade e mortalidade da HIV/ AIDS no mundo ${ }^{6,7}$. Fato este que vem possibilitando uma perspectiva de erradicação desta epidemia nos grandes centros urbanos, até 2030. Isto será possível devido a mobilização da comunidade científica, comitês e organizações de saúde, que passaram a estabelecer metas, assim como o próprio empenho da sociedade. Acreditase que até $2020,90 \%$ dos contaminados conhecerão seu estado sorológico, $90 \%$ dos diagnosticados receberão tratamento com TARV e 90\% terão níveis indetectáveis de HIV, com supressão viral ${ }^{8}$.

Entretanto, o entusiasmo inicial gerado pelos resultados satisfatórios em relação ao uso da TARV contrapõe-se ao uso prolongado. Vários estudos vêm alertando sobre o surgimento de efeitos metabólicos adversos, incluindo manifestações neurológicas como polineuropatias, aumento do risco da toxicidade mitocondrial, mudanças na distribuição da gordura corporal, resistência à insulina e intolerância à glicose, diabetes mellitus tipo II, dislipidemia, acidemia láctica e osteopenia e, ainda, o aparecimento de manifestações neurológicas (alterações mentais, distúrbios da marcha, fraqueza muscular, tremor postural, alterações na coordenação, hiperreflexia, hipertonia e alterações sensoriais) $)^{9,10,11}$.

Outro fator relevante, já discutido, é a neurotoxicidade medicamentosa das TARV, pois estas podem provocar toxicidade mitrocondrial aumentando o risco de promover estresse oxidativo, comprometendo o sistema nervoso, e mais recentemente tem sido assunto de discussão o desenvolvimento de anormalidades metabólicas decorrentes da exposição prolongada à TARV. Assim, questiona-se sua ação como fator contribuidor para o desenvolvimento de disfunções de ordem neuromusculares, com consequente prejuízo ao equilíbrio postural ${ }^{7,12}$.

Para a avaliação de déficits motores, existem inúmeros testes e escalas de avaliações que identificam tais achados. Segundo Botha e Pienaarr ${ }^{13}$, as alterações na coordenação motora de crianças/adolescentes vivendo com HIV são evidenciadas de forma progressiva com o decorrer da idade, de maneira que esta população apresenta dificuldade para a realização de tarefas mais complexas, necessitando do uso de grandes grupos musculares. Desta forma, as habilidades corporais do sistema motor grosso apresentam maiores declínios de funções como a marcha, para os ajustes posturais necessários para o equilíbrio, para as habilidades motoras-perceptivas e, ainda, as alterações no funcionamento muscular que envolve a redução de força muscular, alterações de tônus e perda da funcionalidade.

O teste Korperkoordination Fur Kinder - (KTK) criado por Kiphard e Schilling ${ }^{14}$, em 1974, tem por objetivo analisar equilíbrio, ritmo, força, lateralidade, velocidade e agilidade por meio de quatro testes específicos, com o objetivo de detectar inicialmente déficits motores. Sua elegibilidade se deu por abranger tarefas motoras compatíveis com o desenvolvimento da coordenação motora desde a infância até a adolescência.

Desta maneira, foi objetivo deste estudo determinar o perfil do desempenho motor de crianças e adolescentes infectadas por via vertical pelo HIV e expostas ao uso de TARV. 


\section{MATERIAIS E MÉTODOS}

Trata-se de um estudo observacional e transversal, no qual participaram 34 crianças e adolescentes, sendo 21 com sorologia positiva para o HIV e exposição prolongada às TARV há mais de um ano (Grupo Experimental - GE) e 13 crianças e adolescentes com sorologia negativa para o HIV, porém expostos a medicação nos períodos pré, peri ou pós-natal (Grupo Controle - GC), de ambos os sexos e com idade de seis a 15 anos. A participação no estudo ocorreu após o devido preenchimento do Termo de Consentimento Livre e Esclarecido e Termo de Assentimento. O estudo foi aprovado pelo comitê de ética e pesquisa da Universidade da Amazônia sob o registro no 364864 .

Como critérios de inclusão do grupo experimental: crianças e adolescentes com diagnóstico clínico e laboratorial de AIDS com contagem de células CD4 $>350$ cells $/ \mu \mathrm{L}$, faixa etária de seis a 15 anos de idade; ambos os sexos; infectadas por transmissão vertical, em utilização da terapia antirretroviral por período igual ou maior a um ano, matriculados na coordenação estadual de doenças sexualmente transmissíveis do Estado do Pará e atendidos na URE-MIA.

Os critérios de inclusão para o grupo controle foram: crianças e adolescentes com diagnóstico clínico e laboratorial de infecção pelo HIV com sorologia negativa, na faixa etária de seis a 15 anos de idade, ambos os sexos, que foram expostas à terapia antirretroviral no período pré, peri ou pós-natal, matriculados na Coordenação Estadual de doenças sexualmente transmissíveis do Estado do Pará e atendidos na URE-MIA.

Os critérios de exclusão para ambos os grupos foram: limitações físicas incapacitantes, deficiência visual ou auditiva e presença de hemoptise.

As crianças de ambos os grupos eram encaminhadas para a clínica de fisioterapia Pulmocenter, situada em Belém-PA, local da coleta de dados.

A coleta de dados iniciou com a anamnese de todos os participantes por meio de ficha de identificação e, em seguida, foi aplicado teste para avaliação da coordenação motora grosseira.

A análise da coordenação motora grosseira foi realizada por meio da bateria de testes KTK. De acordo com Gorla et al. ${ }^{15}$, esse teste foi construído, a priori, para determinação da situação de desenvolvimento do domínio corporal de crianças portadoras de deficiência. Atualmente observa-se sua utilização em diversos grupos populacionais, inclusive de crianças saudáveis. Ribeiro et al. ${ }^{16}$ realizaram estudo para averiguar a existência de padrões de referência do KTK para coordenação motora de crianças brasileiras e atestaram ser um instrumento adequado para avaliação do desempenho motor coordenativo, que pode ser aplicado em diferentes populações de crianças saudáveis, portadoras de deficiências, com hipermobilidade articular, prematuras e aquelas que apresentam sobrepeso/obesidade, desta maneira mostrando ser um bom instrumento para a análise do perfil motor grosseiro.

O teste é composto por quatro tarefas: 1) trave de equilíbrio; 2) saltos laterais; 3) saltos monopedais; 4) transferências sobre plataforma. Trata-se de uma bateria homogênea, que utiliza as mesmas tarefas para várias idades. Para isso, os conteúdos das tarefas estão organizados em tarefas que apresentam graus de dificuldades variáveis, que aumentam conforme a idade. A diferenciação por idades segue os seguintes critérios como: 1) aumento da altura ou distância; 2) aumento da velocidade; 3) maior precisão na execução, medida, por exemplo, em função do maior número de acertos num determinado número de tentativas. Para determinar os quocientes motores (QM), obtidos pelas crianças, utilizaram-se tabelas normativas, tal como proposto por Gorla et al. ${ }^{15}$. A confiabilidade do teste ao realizar a bateria completa, ou seja, todas as tarefas é de 0,90, demonstrando fidedignidade.

Após a realização das quatro tarefas foi feito o somatório dos quatro $\mathrm{QM}$, obtendo um escore total referente ao quociente motor global (QMG), que pode ser apresentado em valores percentuais ou absolutos por meio de uma tabela normativa conforme a idade de crianças e adolescentes, permitindo com isso classificar, por cronologia, a população estudada, segundo o nível de desenvolvimento coordenativo: 1) perturbações da coordenação; 2) insuficiência coordenativa; 3) coordenação normal; 4) coordenação boa; 5) coordenação alta. A bateria KTK permite, portanto, dois tipos de análise dos resultados: por tarefa ou pelo valor global do QM. A análise da classificação da coordenação motora no presente estudo foi realizada através da divisão em dois grupos de coordenação, considerando as categorias coordenação motora normal, boa e alta como coordenação normal e o outro grupo com perturbações e insuficiência na coordenação, como coordenação alterada.

A análise de dados foi realizada por meio do programa Bioestat $5.0^{\circledR}$. Por tratar-se de um instrumento de quantificação, cuja distribuição é não paramétrica. Vez que os dados não foram expressos de forma contínua, adotou-se as medidas de mediana e intervalo interquartil (25-75\%), utilizando-se o teste Mann-Whitney para amostras independentes. Estabeleceu-se o nível de significância de 5\% para rejeição de hipótese nula. Para a classificação da coordenação motora divida em coordenação motora normal e alterada foram divididas e expressas em valores numéricos em cada grupo, utilizando-se o teste Exato de Fisher. 


\section{RESULTADOS}

A amostra foi constituída por 34 crianças e adolescentes. GE com $n=21$ no e GC com $n=13$. O GE contou com $57,1 \%$ de indivíduos do sexo masculino, $42,8 \%$ de indivíduos do

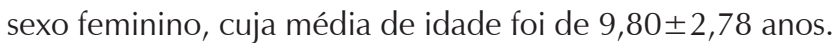
Já o GC apresentou total de $53,8 \%$ de indivíduos do sexo feminino e $46,1 \%$ de indivíduos do sexo masculino com média de idade de $8,07 \pm 2,21$ anos. Os dados dos testes motores da bateria KTK estão expressos com seus respectivos QM, escores, QMG e a classificação da coordenação motora na tabela de caracterização da amostra (Tabela 1).

A Figura 1 mostra a análise feita pelo teste Mann-Whitney entre o GC e o GE dos QM 1 e 4, referentes à investigação das tarefas-trave de equilíbrio e transferências sobre plataforma, respectivamente. Observa-se que o GC apresentou melhor desempenho em relação ao $\mathrm{GE}$, como descritos a seguir pela medianas e intervalos interquatis: QM1 [GC $=102$ $(96,5-130,5), \mathrm{GE}=91(83,5-103), p=0,003)] ; \mathrm{QM} 4[\mathrm{GC}=115$ $(86-129,5), \mathrm{GE}=84(73,5-103), p=0,007)]$.

Para os QM 2 e 3, referentes às tarefas dos saltos laterais e saltos monopedais, não houve diferença com significância estatística entre os grupos. A somatória dos valores obtidos de QM1, QM2, QM3 e QM4 gerou o valor de QMG, demonstrado na Figura 2. Houve diferença estatisticamente significante para o QMG $(p=0,027)$ e observa-se para os seguintes valores $\mathrm{GC}=378(346-429,5)$ e $\mathrm{GE}=333$ (292-361,5).

Tabela 1. Caracterização da amostra.

\begin{tabular}{lccc}
\hline Variáveis & GC & GE & p \\
\cline { 1 - 2 } Sexo (F/M) & Média \pm DP & Média \pm DP & \\
Idade (ANOS) & $7 F / 6 M$ & $9 F / 12 ~ M$ & \\
Variáveis* & $8 \pm 2,21$ & $9 \pm 2,78$ & \\
KTK (qm1) & Mediana (IQ 25-75\%) & Mediana (IQ 25-75\%) & \\
KTK (qm2) & $102(96,5-130,5)$ & $91(83,5-103)$ & 0,003 \\
KTK (qm3) & $85(73-98)$ & $77(55,5-88,5)$ & 0,09 \\
KTK (qm4) & $85(72-90,5)$ & $74(60-89)$ & 0,11 \\
Escore & $115(86-129,5)$ & $84(73,5-103)$ & 0,007 \\
QMG & $93(82-109,5)$ & $78(65-89)$ & 0,003 \\
Classificação da coordenação** & $378(346-429,5)$ & $333(292-361,5)$ & 0,027 \\
Normal & $\mathbf{n}$ & $\mathbf{n}$ & \\
Alterado & 9 & 7 & 0,04 \\
\hline
\end{tabular}

*Teste Mann-Whitney, $p<0,05$. ** Teste Exato de Fisher, $p<0,05$.

GC: Grupo Controle; GE: Grupo Experimental; F: feminino; M: masculino; DP: desvio padrão; IQ: intervalo interquartil; KTK: testes Korperkoordinationstest fur Kinder; qm1: quociente motor 1; qm2: quociente motor 2; qm3: quociente motor 3; qm4: quociente motor 4; QMG: quociente motor global.

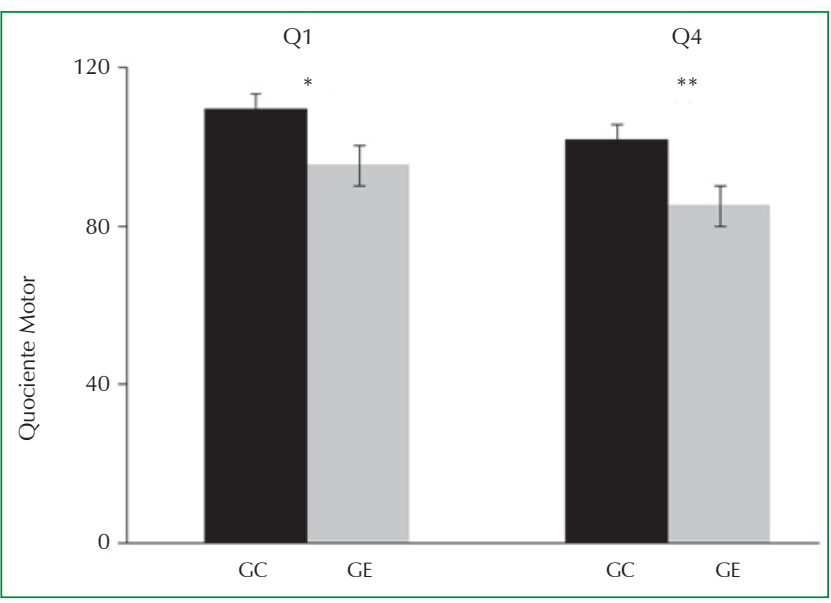

Figura 1. Resultados do teste Mann-Whitney sobre o quociente motor 1 e 4 referentes a análise das tarefas trave de equilíbrio e transferências sobre plataforma, respectivamente, do GC e do GE. GC: Grupo Controle; GE: Grupo Experimental. * $p=0,003 ; * * p=0,007$.

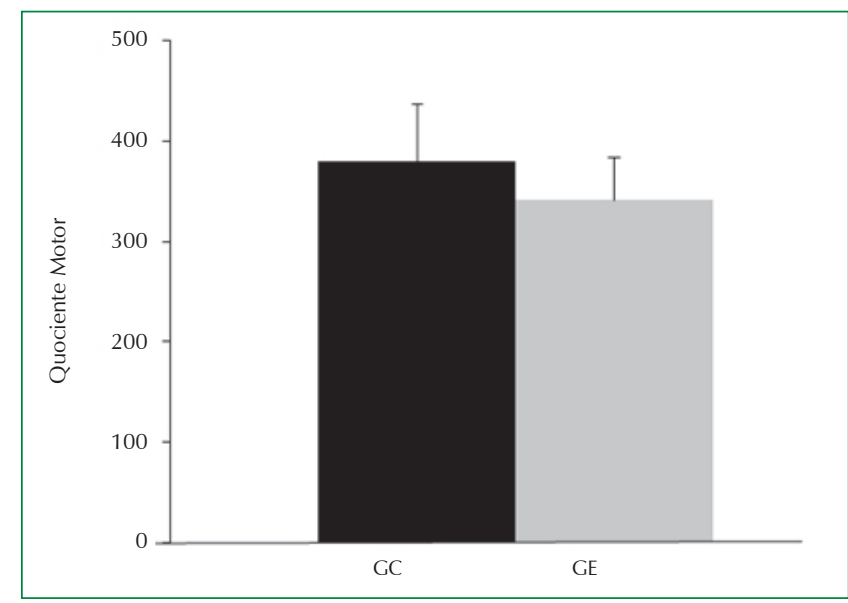

Figura 2. Análise do quociente motor global do GC e do GE. GC: Grupo Controle; GE: Grupo Experimental. $p=0,027$. 
A relação do QMG com a idade cronológica da criança origina um escore númerico que classifica a criança conforme a coordenação motora. Assim, a criança pode ser classificada conforme mostra a Figura 3. Observa-se que o GC obteve maior escore [GC $=93(82-109,5)]$, enquanto o GE apresentou menor [GE=78 (65-89)]; foi encontrada diferença estatística entre os grupos $(p=0,003)$.

A análise dos dados da classificação motora foi dividida em coordenação normal, que corresponde à extratificação de coordenação alta, boa e normal, e a coordenação alterada, que corresponde às pertubações na coordenação e insuficiência na coordenação. Assim, na Figura 4, observa-se que o GE obteve maior número de indivíduos com coordenação motora alterada e o GC como coordenação motora normal, com diferença estatística entre os grupos $(p=0,04)$. A classificação foi realizada por meio do Teste Exato de Fisher, admitindo $p<0,005$.

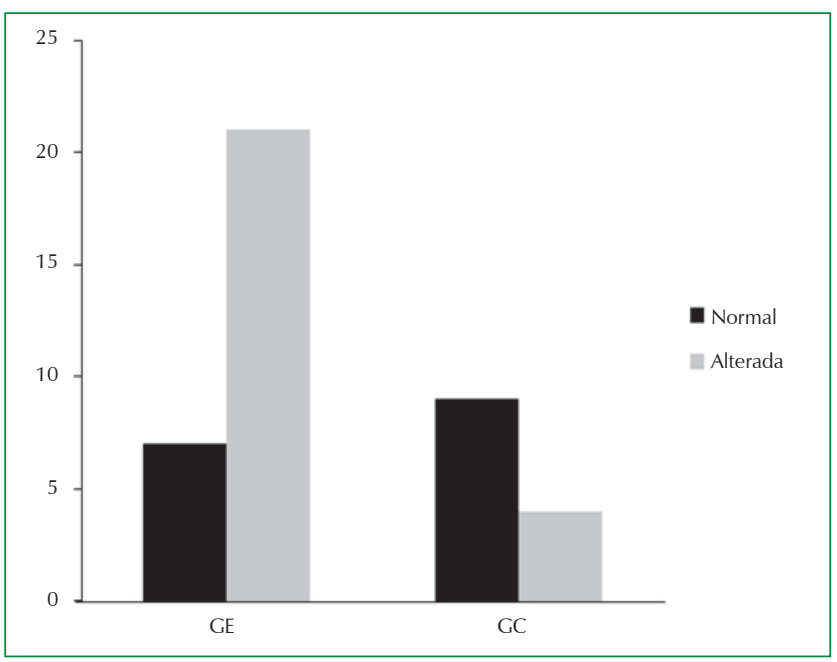

Figura 3. Classificação motora dos grupos amostrais. GC: Grupo Controle; GE: Grupo Experimental. $p=0,003$.

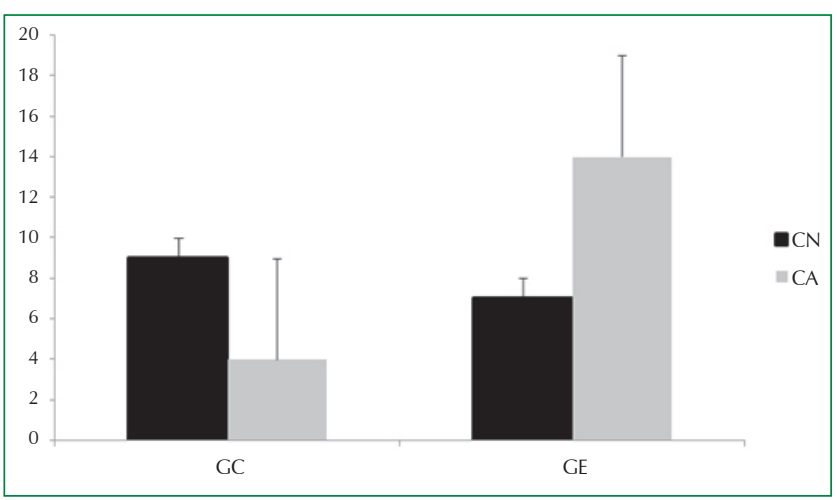

Figura 4. Análise da coordenação motora dos GE e GC pelo teste Exato de Fisher. GC: Grupo Controle; GE: Grupo Experimental; CN: Coordenação Normal; CA: Coordenação Motora Alterada

\section{DISCUSSÃO}

A introdução das drogas antirretrovirais elevou consideravelmente a expectativa de vida de pessoas que vivem com AIDS, porém o seu uso prolongado vem levantando discussões acerca dos seus efeitos adversos. Apesar das evidências das repercussões metabólicas e cardíacas do uso prolongado dessas medicações na população adultaa ${ }^{17}$, pouco se sabe sobre os prejuízos causados à população infantil, que ainda utiliza clinicamente, sem forte evidência, durante as fases de desenvolvimento neuropsicomotor. Portanto, acredita-se que crianças com AIDS e em uso prolongado de TARV encontram-se em condição de maior vulnerabilidade para alterações precoces no sistema sensório-motor. Assim, este estudo objetivou determinar o perfil do desempenho motor de crianças e adolescentes infectadas por via vertical pelo HIV e expostas ao uso de TARV.

Nesta pesquisa, as crianças do GC apresentavam faixa etária mais baixa, que as do GE. Na avaliação intergrupos percebeu-se que GE apresentou maior alteração na coordenação motora grossa com déficits para as tarefas de equilíbrio e lateralidade em relação a tempo/espaço quando comparado ao GC. Na avaliação intragrupo, as crianças mais velhas do GE apresentaram pior desempenho motor em comparação com as crianças mais jovens. Esse fato é contrário ao desenvolvimento motor progressivo com a idade. É provável que tais alterações no sistema motor apresentem forte relação com a perda de força muscular global em crianças em uso extensivo de TARV ${ }^{18,19}$, que porventura tenha interferido nas crianças do GE a desempenharem corretamente as tarefas que requeiram grandes recrutamentos musculares, como saltar e ainda subir escadas ${ }^{20}$. Desse modo, foi possível encontrar crianças em idade avançada soropositivas, que possuem atraso no desenvolvimento motor grosso ${ }^{21,22}$. Para a American Psychiatric Association ${ }^{23}$, em crianças saudáveis não parece haver causa estabelecida para o atraso no desenvolvimento, embora possa ter forte relação com disfunções do sistema nervoso central.

Segundo Rie et al. ${ }^{24}$ após o sistema imunológico, o sistema nervoso é o mais comum sítio de comprometimento em indivíduos com infecção pelo HIV. Já nos primeiros anos de vida das crianças infectadas pelo vírus HIV é possível detectar atrasos no desenvolvimento mental e motor, revelados por alteração na coordenação motora, no tônus muscular e nos reflexos posturais. No nosso grupo, as crianças com HIV mais velhas, apresentam maior tempo vivendo com o HIV, assim como maior exposição aos efeitos colaterais do uso prolongado das TARV.

Silva et al. ${ }^{25}$ estudaram os transtornos do desenvolvimento da coordenação, encontraram uma prevalência maior de 
atraso na aptidão motora em crianças saudáveis e idades mais avançadas, por isso a detecção precoce de atrasos no desenvolvimento neuropsicomotor de crianças soropositiva deve ser o mais antecipado possível, para que a intervenção também seja precoce, com tratamento adequado que permita melhor qualidade de vida ${ }^{12}$.

Neste estudo, foi possível identificar resultados estatisticamente significantes para as tarefas em trave, de equilíbrio e lateralidade, sendo o desempenho do GE inferior ao do grupo GC. Ruel et al. ${ }^{26}$ observaram que crianças de seis a 12 anos de idade infectadas pelo HIV apresentaram déficit de equilíbrio, diminuição de força, déficits de coordenação de membros superiores, alterações na velocidade/agilidade.

Gorla et al. ${ }^{27}$ estudaram a coordenação motora de 283 crianças brasileiras saudáveis e observaram que crianças de seis a oito anos de idade apresentaram um desempenho motor melhor nas tarefas da trave de equilíbrio e transferência, concluindo que, com o avançar da idade, espera-se que crianças consigam obter autonomia para o desenvolvimento de suas experiências motoras, todavia ainda é possível encontrar uma prevalência de 5 a $10 \%$ destas que apresentaram déficits na coordenação motora. Contudo, Guedes $^{28}$ relata que, para possuir noções da coordenação, é importante que haja um acompanhamento dos indicadores do desempenho motor que possam contribuir de forma decisiva para o desenvolvimento de crianças e adolescentes, permitindo assim um incentivo à prática de atividades físicas durante toda a vida.

As crianças e adolescentes do presente estudo situavamse em condição de vulnerabilidade social, econômica e biológica. A geografia da região amazônica dificulta o acesso aos serviços de saúde para um bom acompanhamento clínico, sendo a maioria dos participantes provenientes de áreas rurais ou ribeirinhas e vivem em estado de pobreza.

É reconhecido que as habilidades motoras são dependentes das experiências ambientais, de forma que quanto mais se praticam atividades, melhores serão os desempenhos e habilidades motoras ${ }^{29}$. Então, não basta apenas analisar quais são os prejuízos para o desenvolvimento motor, mas também é necessária uma análise de quais são os fatores que ocasionam esses déficits, tais quais, condição ambiental, baixo poder aquisitivo, baixa escolaridade, dificuldade ao incentivo, à prática de esportes e atividades físicas e/ou condições sociobiológicas como, por exemplo, condições do nascimento, podem contribuir para o surgimento de déficits motores. E com a descoberta dessas alterações atípicas do desenvolvimento motor grosso é viável que ocorram intervenções feitas por equipe de saúde interdisciplinar, dentre eles o fisioterapeuta, para que ocorra a recuperação das habilidades motoras comprometidas ${ }^{30}$.
Este estudo apresentou como limitações o tamanho reduzido da amostra, necessitando de estudos com um número maior. A escassez de estudos avaliando o desempenho motor grosso na população de crianças e adolescentes portadores da AIDS dificultou comparar outros estudos com essa população, assim como o instrumento de avaliação proposto na discussão. Os estudos com a bateria de teste KTK, em suma, apresentam-se em sua maioria nos bancos de dados com a população saudável, restringindo a algumas outras síndromes. Não foi investigado sobre prática esportiva e realização de alguma atividade física, além do diagnóstico e média da exposição da terapia antirretroviral. Sendo assim, pode influenciar nos resultados e associações na discussão.

Desta maneira, na amostra estudada foi identificado que crianças soropositivas expostas às TARV apresentaram pior desempenho motor em relação às crianças com sorodiagnóstico negativo. Foi verificado um pior desempenho para tarefas de equilíbrio e lateralidade, assim como o desempenho motor global foi inferior nesse grupo. Esses achados apontam para anormalidades na coordenação motora grosseira em crianças $\mathrm{HIV}^{+}$assintomáticas para disfunções neurológicas. Contudo, sabe-se que o aprendizado motor requer treinamento e aprendizagem, para que o desenvolvimento das tarefas seja realizado com agilidade e destreza. Dessa forma, a intervenção fisioterapêutica preventiva se faz importante para minimizar danos ao equilíbrio.

Além das análises precoces, recomenda-se possibilitar medidas preventivas e educativas em escolares, para conscientizar crianças e adolescentes da importância de a adesão à prática de atividade física regular e mudanças nos hábitos de vida, com a finalidade de estimular para que estas possam atingir um desempenho motor pleno. $\mathrm{O}$ conhecimento do curso clínico da patologia, dos avanços na terapia medicamentosa, assim como os efeitos adversos da terapia de longa duração deve ser extensivamente estudado, para que se possa ter futuras gerações vivendo com o HIV, mas com boa saúde física e qualidade de vida.

\section{REFERÊNCIAS}

1. Brasil. Ministério da Saúde. Boletim Epidemiológico - AIDS e DST [Internet]. Brasília: Ministério da Saúde. [atualizado em jul. 2009; citado em ago. 2015]. Disponível em: http://www.aids.gov.br/ publicacao/2009/boletim-epidemiologico-aids-2009

2. Organização Mundial da Saúde. Global Report: Unaids Report on the Global AIDS Epidemic 2013 [Internet]. Geneva: Unaids. [atualizado nov. 2013; citado em dez. 2015]. Disponível em: http:// www.unaids.org/sites/default/files/media_asset/UNAIDS_Global_ Report_2013_en_1.pdf

3. Brasil. Ministério da Saúde. Boletim Epidemiológico HIV/AIDS [Internet] 
4. Brasília: Ministério da Saúde. [atualizado em jun. 2015; citado em jan. 2016]. Disponível em: http://www.aids.gov.br/sites/default/ files/anexos/publicacao/2015/58534/boletim_aids_11_2015_web_ pdf_19105.pdf

5. Melo MC de, Ferraz RO, Nascimento JL do, Donalisio MR. Incidência e mortalidade por AIDS em crianças e adolescentes: desafios na região sul do Brasil. Ciênc Saúde Coletiva. 2016;21:3889-98. https://doi.org/10.1590/1413-812320152112.11262015

6. Brasil. Ministério da Saúde. Boletim Epidemiológico HIV-AIDS [Internet]. Brasília: Ministério da Saúde. [atualizado em jun. 2014; citado em jul. 2015]. Disponível em: http://www.aids.gov.br/sites/ default/files/anexos/publicacao/2014/56677/boletim_2014_final_ pdf_15565.pdf.

7. Battistini TRB, Sarni ROS, de Souza FIS, Pitta TS, Fernandes AP, Hix S, Fonseca FL, Tardini PC, dos Santos VP, Lopez FA. Lipodystrophy, lipid profile changes, and low serum retinol and carotenoid levels in children and adolescents with acquired immunodeficiency syndrome. Nutrition. 2010;26:612-6. https://doi.org/10.1016/j. nut.2009.06.024

8. Woods SP, Moore DJ, Weber E, Grant I. Cognitive neuropsycology of HIV-associated neurocognitive disorders. Neuropsychol Rev. 2009;19:152-68. https://doi.org/10.1007/s11065-009-9102-5

9. Programa Conjunto das Nações Unidas sobre o HIV/AIDS (Unaids). Ambitious Treatment Targets: Writing the final chapter of the AIDS epidemic [Internet]. Geneva; 2014 (atualizado em mar. 2014; citado em ago. 2015). Disponível em: http://www.unaids.org/sites/default/ files/media_asset/JC2670_UNAIDS_Treatment_Targets_en.pdf

10. Fabiano V, Giacomet V, Viganò A, Bedogni G, Stucchi S, Cococcioni L, Mora S, Zuccotti GV. Long-term Body composition and metabolic changes in HIV-infected children switched from stavudine to tenofovir and from protease inhibitors to efavirenz. Eur J Pediatr. 2013;172:1089-96. https://doi.org/10.1007/s00431-013-2018-3

11. Haase VG, Nicolau NC, Viana VN, Barreto GV, Pinto JA. Executive function and processing speed in brazilian HIV-infected children and adolescents. Dement Neuropsychol. 2014;8:32-9. https://doi. org/10.1590/S1980-57642014DN81000006

12. Gonzalez- Duarte A, Cikurel K, Simpson DM. Selected neurologic complications of HIV and antiretroviral therapy. The PRN Notebook. $2004 ; 1: 24-9$.

13. Capelo, AV, Sá CAM, Rubini NP, Kalil RS, Miranda E. Impacto da Neuro-AIDS na infância. DST J Bras Doenças Sex Transm. 2006;18:259-62.

14. Botha JA, Pienaar AE. The motor development of 2 to 6 year old children infected with HIV. Sajr Sper. 2008;30(2):39-51. https://doi. org/10.4314/sajrs.v30i2.25982

15. Kiphard EJ, Schilling VF. Köper-koordinations-test für kinder: KTK. Weinhein: Beltz Test Gmbh; 1974.

16. Gorla JI, Araújo PF, Rodriguez JL. Avaliação motora em educação física adaptada: teste KTK. 2aㅡ ed. São Paulo: Phorte; 2009.

17. Ribeiro AC, David AC, Barbacena MM, Rodrigues ML, França NM. Teste de coordenação corporal para crianças (KTK): aplicações e estudos normativos. Motricidade. 2012;8:40-51. https://doi. org/10.6063/motricidade.8(3).1155

18. Fortuny C, Deya-Martinez A, Chiappini E, Galli L, Martino M de, Noguera-Julian A. Metabolic and renal adverse effects of antiretroviral therapy in hiv-infected children and adolescents. Pediatr Infect Dis J. 2015;34:S36-S43. https://doi.org/10.1097/ INF.0000000000000663

19. Martino M, Tovo PA, Balducci M, Galli L, Gabiano C, Rezza G, Pezzotti P. Reduction in mortality with availability of antiretroviral therapy for children with perinatal HIV-1 infection. Italian Register for HIV Infection in Children and the Italian National AIDS Registry. JAMA. 2000;284:190-7. https://doi.org/10.1001/jama.284.2.190

20. Somarriba G, Lopez-Mitnik G, Ludwig DA, Neri D, Schaefer N, Lipshultz SE, Scott GB, Miller TL. Physical fitness in children infected with the human immunodeficiency virus: associations with highly active antiretroviral therapy. AIDS Res Hum Retroviruses. 2013,29:112-20. https://doi.org/10.1089/aid.2012.0047

21. Baillieu N, Potterton J. The extent of delay of language, motor, and cognitive development in HIV-Positive infants. JNPT. 2008,32: 118-21. https://doi.org/10.1097/NPT.0b013e3181846232

22. Lingam R, Hunt L, Golding J, Jongmans M, Emond A. Prevalence of developmental coordination disorder using the dsm-iv at 7 years of age: a UK population-based study. Pediatrics. 2009;123:693-701. https://doi.org/10.1542/peds.2008-1770

23. Kourtessis T, Thomaidou E, Liveri-Kantere A, Michalopoulou M, Kourteiss K, Kioumourtzoglou E. Prevalence of developmental coordination disorder among greek children with learning disabilities. Eur Psychomot J. 2008;1:10-7.

24. American Psychiatric Association (APA). Diagnostic and statistical manual of mental disorders (DSM-V) [versão online]. Arlington: APA; 2010 [citado em jan. 2016]. Disponível em: http://dsm.psychiatryonline.org/doi/book/10.1176/appi. books.9780890425596

25. Van Rie A, Mupuala A, Dow A. Impact of the HIV/AIDS epidemic on the neurodevelopment of preschool-aged children in Kinshasa, Democratic Republic of the Congo. Pediatrics. 2008;122:1-12. https://doi.org/10.1542/peds.2007-2558

26. Silva JS, Beltrame TS. Indicativo de transtorno do desenvolvimento da coordenação de escolares com idade entre 7 a 10 anos. Rev Bras Cienc Esporte. 2013;35:3-14. https://doi.org/10.1590/S010132892013000100002

27. Ruel TD, Boivin, MJ, Boal HE, Bangirana P, Charlebois E, Havlir DV. Neurocognitive and motor deficits in HIV-infected Ugandan children with high CD4 cell counts. Clin Infect Dis. 2012;54: 1001-9. https://doi.org/10.1093/cid/cir1037

28. Gorla JI, Duarte E, Montagner PC. Avaliação da coordenação motora de escolares da área urbana do município de Umuarama-PR Brasil. R Bras Ci e Mov. 2008;16:57-65.

29. Guedes DP. Implicações associadas ao acompanhamento do desempenho motor de crianças e adolescentes. Rev Bras Educ Fis Esp. 2007; 21:37-60.

30. Catenassi FZ, Marques I, Bastos CB, Basso L, Ronque ERV, Gerage AM. Relação entre índice de massa corporal e habilidade motora grossa em crianças de quatro a seis anos. Rev Bras Med Esporte. 2007;13:227-30. https://doi.org/10.1590/S1517-8692 2007000400003

31. Willrich A, Azevedo CCF, Fernandes JO. Desenvolvimento motor na infância: influência dos fatores de risco e programas de intervenção. Rev Neurocienc. 2009; 17:51-6. 